\title{
In Vivo Delivery of Cas9 Ribonucleoprotein and Donor DNA with Gold Nanoparticles
}

\author{
Niren Murthy \\ University of California at Berkeley \\ USA
}

Cas9 based therapeutics have the potential to revolutionize the treatment of genetic diseases because of their ability to generate homologous DNA recombination (HDR) and correct DNA mutations. However, viral gene therapy is currently the only delivery technology available for generating HDR in vivo with Cas9, and is challenging to bring into clinical trials because of off-target DNA damage and immunogenicity. In this presentation, I will describe a non-viral Cas 9 delivery vehicle, termed CRISPR-Gold, which can induce HDR in vivo by directly delivering Cas9 protein, gRNA, and donor DNA. CRISPR-Gold is composed of gold nanoparticles assembled with the Cas9/gRNA ribonucleoprotein (RNP) complex, donor DNA, and an endosomal disruptive polymer. We have been able to demonstrate that CRISPR-Gold can correct the DNA mutation that causes Duchenne muscular dystrophy (DMD) in mdx mice via HDR, with an efficiency of 5.4\% after an intramuscular injection and with minimal levels off-target DNA damage. In addition, we demonstrate that CRISPR-Gold can improve muscle strength and lower tissue fibrosis in mdx mice. CRISPR-Gold is the first example of a DMD therapeutic that can correct the mutated dystrophin protein back to its wild type sequence, and has tremendous potential for treating DMD and other genetic diseases caused by single base pair mutations. 